\title{
Molecularly imprinted polymers coupled to mass spectrometric detection for metallothionein sensing
}

\author{
VANĚČKOVÁ, T.; POMPEIANO VANÍČKOVÁ, L.; TVRDOŇOVÁ, M.; POMORSKI, A.;
}

KRĘŻEL, A.; VACULOVIČ, T.; KANICKÝ, V.; VACULOVIČOVÁ, M.; ADAM, V.

\section{Talanta}

2019, vol. 198, June 2019, pp. 224-229

ISSN: 0039-9140

DOl: https://doi.org/10.1016/j.talanta.2019.01.089

Accepted manuscript

(C) 2018. This manuscript version is made available under the CC-BY-NC-ND 4.0 license (http://creativecommons.org/licenses/by-nc-nd/4.0/),

doi: https://doi.org/10.1016/j.talanta.2019.01.089

Final version available from

https://www.sciencedirect.com/science/article/pii/S0039914019301109 


\section{Molecularly imprinted polymers coupled to mass spectrometric detection \\ for metallothionein sensing}

Tereza Vaneckova ${ }^{1,2}$, Lucie Pompeiano Vanickova ${ }^{1,2}$, Michaela Tvrdonova $^{3}$, Adam Pomorski ${ }^{4}$, Artur Krężel ${ }^{4}$, Tomas Vaculovic ${ }^{3,5}$, Viktor Kanicky ${ }^{3,5}$, Marketa Vaculovicova ${ }^{1,2}$, Vojtech Adam ${ }^{1,2 *}$

${ }^{1}$ Department of Chemistry and Biochemistry, Mendel University in Brno, Zemedelska 1, CZ61300 Brno, Czech Republic

${ }^{2}$ Central European Institute of Technology, Brno University of Technology, Purkynova 123, CZ-612 00 Brno, Czech Republic

${ }^{3}$ Department of Chemistry, Faculty of Science, Masaryk University, Kamenice 753/5, CZ-625 OO Brno, Czech Republic

${ }^{4}$ Department of Chemical Biology, Faculty of Biotechnology, University of Wroclaw, JoliotCurie 14a, 50-383 Wroctaw, Poland

${ }^{5}$ Central European Institute of Technology, Masaryk University, Kamenice 753/5, CZ-625 00 Brno, Czech Republic

* Corresponding author: Vojtech Adam, Department of Chemistry and Biochemistry, Mendel University in Brno, Zemedelska 1, CZ-613 00 Brno, Czech Republic; E-mail: vojtech.adam@mendelu.cz; phone: +420-5-4513-3350; fax: +420-5-4521-2044 


\begin{abstract}
We report a facile method for detection of metallothionein (MT), a promising clinically relevant biomarker, in spiked plasma samples. This method, for the first time, integrates molecularly imprinted polymers as purification/pretreatment step with matrix assisted plasma desorption/ionization time-of-flight mass spectrometric detection and with laser ablation inductively coupled plasma mass spectrometry for analysis of MTs. The prepared MTimprinted polydopamine layer showed high binding capacity and specific recognition properties toward the template. Optimal monomer (dopamine) concentration was found to be $16 \mathrm{mM}$ of dopamine. This experimental setup allows to measure $\mu \mathrm{M}$ concentrations of $\mathrm{MT}$ that are present in blood as this can be used for clinical studies recognizing MT as marker of various diseases including tumour one. Presented approach not only provides fast sample throughput but also avoids the limitations of methods based on use of antibodies (e.g. high price, cross-reactivity, limited availability in some cases, etc.).
\end{abstract}

\title{
Keywords
}

Metallothionein; molecularly imprinted polymers; polydopamine; MALDI-TOF-MS; LAICP-MS 


\section{Introduction}

Metallothioneins (MTs) are low molecular mass $(<7 \mathrm{kDa})$, cysteine-rich proteins, ubiquitously present in practically all eukaryotes [1-3]. MTs perform a wide range of functions in an organism including essential metal homeostasis, i.e. $\mathrm{Zn}^{2+}$ and $\mathrm{Cu}^{+}$, heavy metal detoxification, i.e. $\mathrm{Cd}^{2+}$, scavenging of reactive oxygen species and regulation of transcription [4-8]. The elevated concentration of MTs has been observed in blood and/or tissues coming from patients with various tumour diseases (e.g., colon, breast, liver, kidney, lung, nasopharynx, ovary, salivary gland, prostate, thyroid, and urinary bladder cancer) as it was reviewed $[9,10]$, demonstrating that MTs are promising oncosuppressors [11-13].

Numerous analytical approaches have been suggested for detection and determination of MTs as reviewed elsewhere [14, 15]. More recently, a method based on the enzyme-linked immunosorbent assay and the real time polymerase chain reaction was used [16]. Another study reported a microfluidic MT electrochemical immunosensor utilizing superparamagnetic agarose beads [17], where a curve fitting approach was be used for voltammograms of various isoforms of MTs [18]. Mass spectrometry coupled with matrix assisted laser desorption/ionization (MALDI) or inductively coupled plasma (ICP) ionization was also used for determination of MTs [19]. Recently, bottom-up mass spectrometry-based approach for human MT isoforms quantification has been developed [20], nevertheless combinations of these types of detection with biological molecule recognition elements are needed.

Molecularly imprinted polymers (MIPs) are biorecognition surfaces with high affinity towards desired template. Named as natural receptor mimics, MIPs are being considered as an alternative to biological receptors, such as enzymes, antibodies or aptamers [21, 22]. Their major advantages cover predictable specific recognition, low cost, ease of preparation, good mechanical/chemical stability, and reusability [23]. Although MIPs have been successfully applied using wide range of small molecules [24], imprinting of biomacromolecules, such as 
proteins, faces challenges. Macromolecular templates have a tendency to adsorb to polymers, where it is not trivial to remove them from the polymer matrix, as they produce heterogeneous sites, and may be sensitive to denaturation or presence of organic solvents, which are usually essential for formation of polymers $[22,25]$. To date, only few studies reported combination of molecular imprinting technology together with MALDI time-of-flight mass spectrometry (MALDI-TOF-MS) [26-33]. However, determination of MT using molecularly imprinting technology combined with mass spectrometric detection (neither MALDI nor ICP) has not been reported yet.

In the present study, we developed an easy method for MT purification from a complex matrix using MT-selective PDA layer combined followed with MALDI-TOF-MS and LAICP-MS detection techniques (Fig. 1). The analytical performance of the sensor was evaluated. The obtained results reveal a new perspective in recognition and separation of this template, which is a potential marker of diseases.

\section{Material and methods}

\section{Materials}

Dopamine hydrochloride, albumin from human serum $(\geq 97 \%)$, Trizma ${ }^{\circledR}$ (TRIS base), and lysozyme from chicken egg white were purchased from Sigma-Aldrich company (St. Louis, MO, USA). Acetic acid (99.8\%) and hydrochloric acid (reagent grade, 35\%) was obtained from PENTA (Chrudim, Czech Republic). Amicon ${ }^{\circledR}$ Ultra $0.5 \mathrm{~mL} 50 \mathrm{~K}$ Centrifugal Filters were purchased from Merck Millipore (Billerica, MA, USA). Tris-HCl buffer $(20 \mathrm{mM}$, pH 8.5) was prepared from Trizma ${ }^{\circledR}$ and hydrochloric acid was used for $\mathrm{pH}$ adjustment. Aqueous solution of acetic acid $(3 \%, v / v)$ was used as a washing buffer. Deionized water used during the experiments was prepared with a Milli-Q water purification system (Millipore, Milford, MA, USA). 


\section{Preparation of MT-imprinted polydopamine layer}

Polydopamine (PDA) MIP was prepared by self-polymerization inspired by works of [34, 35]. In brief, dopamine was dissolved in $1 \mathrm{~mL}$ Tris- $\mathrm{HCl}$ buffer $(20 \mathrm{mM}, \mathrm{pH} 8.5)$. Template molecules of MT-1, MT-3 or lysozyme, for initial optimization, were then mixed with the stock solution of DA at 1:1 (v/v) ratio. The target concentration was varied depending on the experiment. Next, $1 \mu \mathrm{L}$ of the polymerization mixture was applied on the MALDI target plate (Bruker MTP AnchorChip 384BC ${ }^{\mathrm{TM}}$ ) or, in case of initial experiments, bottom of the 96 wellplate and let to polymerize and dry at the room temperature for $24 \mathrm{~h}$. Subsequently, the prepared polymeric layer was overlaid with a sample solution and the incubation was carried for 1 hour at room temperature. The resulting self-assembled polymer was then washed with $3 \%$ acetic acid to remove the bound MT or lysozyme. Final wash was performed with Milli-Q water. Control, non-imprinted polymer (NIP), was prepared under the same conditions without adding the template (MT/lysozyme). Each of the analysed polymers was prepared in triplicate.

\section{Sample preparation}

\section{Standard solutions}

The coding sequences of human metallothionein-1 (MT-1) (UniProt:P13640-2) and metallothionein-3 (MT-3) (UniProt:P25713-1) were purchased from Genscript (Piscataway, NJ, USA) and inserted into the pTYB21 vector (New England Biolabs, UK). Prepared plasmid transformed into BL21(DE3)pLysS E. coli cells. Protein production and purification was conducted as previously stated [36]. The obtained recombinant protein MT-1 used in the MALDI-MS experiments had seven bound $\mathrm{Zn}(\mathrm{II})$ ions. For LA-ICP-MS experiments were prepared MT-1 with seven bound Cd(II) ions and MT-3 with seven bound $\mathrm{Zn}(\mathrm{II})$ ions. 


\section{Plasma sample preparation}

Whole blood was collected from a healthy volunteer and then the whole blood was centrifuged for $10 \mathrm{~min}$ at $2000 \mathrm{rcf}$ and the plasma was further centrifuged for $30 \mathrm{~min}$ at 22000 rcf. The plasma was diluted $50 \times$ with Tris- $\mathrm{HCl}$ buffer $(20 \mathrm{mM}, \mathrm{pH} 8.5)$ and spiked with MT-1 to its final concentration of $5 \mu \mathrm{M}$. All subjects gave their informed consent for inclusion, before participating in the study. The study was conducted in accordance with the Declaration of Helsinki and the protocol was approved by the Ethics Committee of Masaryk University.

\section{MALDI-TOF-MS}

MIP/NIP were analysed using MALDI-TOF-MS (ultrafleXtreme instrument, Bruker Daltonik $\mathrm{GmbH}$, Bremen Germany) equipped with a laser (operating at wavelength of $355 \mathrm{~nm}$ with an accelerating voltage of $25 \mathrm{kV}$, a maximum energy of $43.2 \mu \mathrm{J}$, and a repetition rate of $2000 \mathrm{~Hz}$ ) in linear positive ion mode for data acquisition. Three different organic matrix solutions were tested, namely $\alpha$-cyano-4-hydroxycinnamic acid, sinapinic acid and 2,5dihydroxybenzoic acid (DHB) (Bruker Daltonik, Bremen, Germany), with DHB diluted in 0,1\% trifluoroacetic acid (Sigma-Aldrich) being considered the optimal solution, since less background was produced in the final spectrum [18]. Matrix $(0.5 \mu \mathrm{L})$ was applied on the prepared MIP and/or NIP polymerized layer (as previously described in section 2.2) and dried under atmospheric pressure and ambient temperature $\left(25^{\circ} \mathrm{C}\right)$. The laser frequency was set to $1000 \mathrm{~Hz}$ and laser energy was optimized prior to each measurement. Calibration was done externally using a protein standard mixture I and II (Bruker Daltonics, Bremen, Germany) in the range of $\mathrm{m} / \mathrm{z} 1-90 \mathrm{kDa}$. A total of 500 spectra were summed for each spot using the Random Walk raster pattern, with no evaluation criteria and were analysed with the Flex Analysis software (Version 3.4). 


\section{Fluorescence spectrometry}

Fluorescence spectrometric measurements were performed using Infinite M200 fluorescence microplate reader (Tecan, Männedorf, $\mathrm{CH})$. Polymerization mixture $(50 \mu \mathrm{L})$ was deposited on the bottom of the well of Corning ${ }^{\circledR} 96$ Well Clear Flat Bottom UV-Transparent Microplate (Corning, NY, USA). Fluorescence emission of lysozyme was recorded at $\lambda_{\mathrm{ex}}=280 \mathrm{~nm}$ and $\lambda_{\mathrm{em}}=330 \mathrm{~nm}$ with gain of the detector set to 100.

\section{LA-ICP-MS}

The analysis of MIP was performed by LA-ICP-MS setup that consists of LA system UP213 (NewWave Research, USA) emitting laser radiation with a wavelength of $213 \mathrm{~nm}$ with a pulse width of $4.2 \mathrm{~ns}$. The ablated material was carried out from an ablation cell by a flow of a He (1.0 1/min) into ICP-MS Agilent 7500CE (Agilent Technologies, Japan) with quadrupole analyzer.

The MIP and NIP were ablated with following ablation parameters: laser beam diameter of $110 \mu \mathrm{m}$, the repetition rate of $10 \mathrm{~Hz}$; laser beam fluence of $6 \mathrm{~J} / \mathrm{cm}^{2}$, the scan speed of $400 \mu \mathrm{m} / \mathrm{s}$ and distance between individual lines of $100 \mu \mathrm{m}$. The analytes MT-1 and MT-3 were monitored by measuring of isotope ${ }^{111} \mathrm{Cd}$ and ${ }^{66} \mathrm{Zn}$, respectively.

\section{Results and discussion}

\section{Optimization of MIPs preparation}

To address limitations of protein imprinting, several strategies have been investigated including metal ion-coordination polymerization, protein epitope approach, and surface imprinting [22, 25, 37]. The latter approach utilizes polydopamine (PDA), one of the most favourable polymers considering its green chemistry status and facile preparation. Dopamine 
(DA), a functional monomer, can form a thin, self-polymerizing film on a wide variety of materials in a weak alkaline environment $(\mathrm{pH}>8)$ [38]. It has been shown that PDA forms thin films by the spontaneous polymerization in the presence of oxygen; however some other polymerization methods involving radicals formation have been developed [39]. DA, commonly involved in human body as a neurotransmitter, is also a small-molecule mimicking the adhesive proteins. Its multifunctional groups and properties of hydrophilicity and biocompatibility make it suitable for imprinting of proteins. It can be self-polymerized under mild conditions (room temperature, $\mathrm{pH}$ 8.0) resulting in formation of an adherent polydopamine film. For example, approach for imprinting proteins using PDA coating of $\mathrm{Fe}_{3} \mathrm{O}_{4}$ nanoparticles has been reported [40]. Xia et al. suggested an approach for protein recognition and separation using PDA-coated molecularly imprinted silica nanoparticles [41]. Therefore, it is believed that polydopamine MIPs are expedient and appropriate materials applicable in the identification of proteins.

The simple oxidative polymerization was employed in this study. For non-covalent imprinting, the optimal ratio of template to functional monomer (T/M) has to be achieved empirically [42]. Therefore, the concentration of monomer (dopamine) was tested in concentrations of $16,32,65$, and $130 \mathrm{mM}$ (data not shown). The functionality of the imprinted polymer was initially tested utilizing lysozyme as a template. The globular glycoprotein lysozyme (Lys) plays and important role in living organisms. Considering its excellent antibacterial property, Lys is widely used in medical and food industry. Thus, the development of an effective purification method for Lys is broadly valuable [43]. Based on the intrinsic fluorescence of Lys, the efficiency of the polymeric layer preparation was evaluated. According to the fluorescence spectrometry measurements, the dopamine concentration of $16 \mathrm{mM}$ was chosen as the most appropriate due to the highest binding yield. 
Subsequently, the effect of template concentration was investigated also by use of Lys in the same manner as optimal dopamine concentration determination. The dopamine concentration of $16 \mathrm{mM}$ was used for polymerization. Concentrations of the template molecules of 9, 17, 35, and $70 \mu \mathrm{M}$ were tested (S1). In our experiment, the evaluation was performed considering the size of the molecule used as a template and was also aimed at use as low amount of protein as possible. The T/M ratio was $0.0005,0.001,0.002$, and 0.004 , even though the $\mathrm{T} / \mathrm{M}$ ratio is suggested to be $0.5-0.25$, however, this depends on the type of the template [42]. As expected, the higher the template concentration, the better signal was obtained due to the fact that more analyte-responsive cavities were formed. Therefore, we selected $70 \mu \mathrm{M}$ as the optimum in further experiments.

\section{MALDI-MS analysis}

DHB was determined as optimal MALDI matrix for MT analysis and therefore it was used in all following MALDI-MS experiments. Consequently, two concentrations of MT-1 (6071.5 $\left.[\mathrm{M}+\mathrm{H}]^{+}\right)$as a template in the DA were tested $(0.2 \mathrm{mM}$ and $0.02 \mathrm{mM}, \mathrm{T} / \mathrm{M}=0.013$ and 0.0013). The data shown in S2A confirm the fact that higher concentration of template increases the signal, as expected.

\section{Template removal}

Numerous approaches to template removal are presented in the literature [44, 45]. Based on these works, $3 \%$ acetic acid $(v / v)$ was chosen for washing. When the PDA imprinted layer (1 $\mu \mathrm{L}$ of polymerization mixture) was washed once with $2 \mu \mathrm{L}$ of $3 \%$ acetic acid $(v / v)$, the vast majority of the template still remained in the PDA layer. Performed experiments revealed that repeated washing (5-times with $2 \mu \mathrm{L}$ of $3 \%$ acetic acid) was required for the sufficient template removal. As shown in S2B, the amount of template molecules remaining on the MIP 
surface after template removal procedure (background signal) was significantly lower compared to the signal obtained after application of the sample (analyte) solution onto the PDA layer. NIP (PDA polymerized in absence of template) was treated exactly the same way as MIP.

Based on this, the final signal results as a comparison between 1) the signals obtained from MIP with applied sample solution (washed with water) with subtracted signal of MIP after template removal (washed with 3\% acetic acid) and 2) the NIP after application of the sample solution (washed with water).

\section{MIP selectivity}

To evaluate the selectivity of prepared MIP, the most abundant protein occurring in blood, albumin, was employed as an interference present in the model sample mixture $\left(\mathrm{c}_{\mathrm{MT}-1}=3 \mu \mathrm{M}\right.$, $\left.\mathrm{c}_{\text {albumin }}=3 \mu \mathrm{M}\right)$. This protein represents a suitable model of interference not only because of its intrinsic presence in blood but also due to its tendency to adsorb on various surfaces that is commonly employed as a blocking agent in immunoassays [46]. As shown in the Fig. 3, metallothionein MIP analysed after application of the model sample solution exhibited only a minor signal of albumin $\left(69368.5[\mathrm{M}+\mathrm{H}]^{+}\right)$, which is shown in the inset in S3, whereas MT $\left(6071.5[\mathrm{M}+\mathrm{H}]^{+}\right)$could be easily identified. PDA exhibits multiple interactions with proteins and weak hydrophilic interactions with salts. Thus the signals of proteins can be improved in spite of contamination by salts [47]. In addition, the MIPs have low nonspecific absorption capacity to non-templates [33]. The above mentioned results validated that the MIPs have specificity toward MT and resulted in a significantly selective enhancement in MS signals of MT. 


\section{Evaluation of different MT concentrations by MALDI-MS}

Based on the literature information, the MT level in blood of healthy persons occurs at the value of $0.5 \mu \mathrm{M}$, however the level in cancer patients ranges from 1.59 to $2.70 \mu \mathrm{M}$ with average and median of 2.12 and $2.07 \mu \mathrm{M}$, respectively [48]. It has to be noticed here that the precise quantification by MALDI-TOF-MS is not trivial and may be problematic even though numerous approaches may be taken to address this issue [49]. Therefore, this work does not aim at exact determination of the MT concentration, but rather targets at development of rapid method to identify the suspicious samples with potentially elevated MT level. Therefore, the $0.6 \mu \mathrm{M}$ concentration of MT occurring in the diseased patients was taken as a threshold value. It should be noted that the samples with elevated MT level provides very high S/N ratio and signal is dependent although non-linear on the concentration of the target. Three MT concentrations $(0.6,1.5$ and $3 \mu \mathrm{M})$ were, thus, tested in order to investigate the possibility of detection of the MT signal. As shown in S4, all of these concentrations were well detected after undergoing the MIP extraction procedure (Fig. 4A) and NIPs were simultaneously performed as a control (Fig. 4B). Therefore, it can be concluded that the method is sensitive and selective enough to indicate the increased level of MT, leading to further quantitative investigation.

\section{Zinc and Cadmium detection in MT by LA-ICP-MS}

As mentioned previously, MALDI-MS it not a method of choice if precise quantification is needed. Therefore, a quantitative mass spectrometric analysis (LA-ICP-MS) was employed to for quantitation of levels of metal ions present in MT. To our best knowledge, this is the first use of LA-ICP-MS in combination with MIP technique. Under physiological conditions metallothioneins bind $\mathrm{Zn}^{2+}$ and $\mathrm{Cu}^{+}$, participating in metabolism of those metal ions [50]. 
Furthermore, cadmium $\left(\mathrm{Cd}^{2+}\right)$ accumulates in MT with exposure and age. For simplification, MT completely saturated with $\mathrm{Zn}^{2+}$ ions or with $\mathrm{Cd}^{2+}$ ions was used in this study.

First, the efficiency of the template removal from MIP by washing with acetic acid was tested. It was found out that the ${ }^{66} \mathrm{Zn}$ on remaining on the MIP surface (and within the PDA layer) after template removal procedure was approximately $1.3 \%$ of the original amount. The nonspecific adsorption of analyte on NIP was observed, as well. Similarly to MALDI-MS experiments, the signal was significantly lower in comparison to MIP, which is extremely beneficial for usage of this protocol as quantification step. To summarize data obtained by LA-ICP-MS we used Fig. 2, where is clearly shown that MIP-MT gave signal significantly differed from others. LA-ICP-MS technique was further applied for distinction of MT-1 (Cd) from MT-3 (Zn) MIP and NIP as demonstrated in Fig. 36.

\section{MT analysis in plasma}

Our MT-MIP MALDI-MS approach has been verified using a real biological sample of human plasma. As illustrated in Fig. 7, no peak of MT-1 was revealed when using NIP. However, after enrichment from diluted human plasma, only a sharp peak of MT-1 (6071.5 $[\mathrm{M}+\mathrm{H}]^{+}$) could be identified on the MT-MIP. Here we have shown that MT-MIPs can capture MT-1 specifically and effectively from complex biosamples. Furthermore, spectrum in the Fig. 4 shows interfering substances $(m / 2,5539.4)$ demonstrating the ability of MS detection to identify the interferents and providing additional information level compared to non-specific detections commonly used with MIP, such as quartz crystal microbalance. Besides precise identification, we further used above mentioned protocol based on LA-ICP-MS and found the level of MT-1 to be $0.05 \mu \mathrm{M}$.

In conclusion, we demonstrated for the first time that MIP may be used as a selective purification step for target metalloproteins for MALDI-MS analyses. Metallothionein- 
molecularly imprinted polymers were successfully prepared by immobilization of the template and self-polymerization of dopamine directly on the MALDI plate and showed good selectivity toward the template protein. Convenient enhancement in MALDI-MS signals was achieved for MT from the spiked protein mixture and plasma sample. Furthermore, LA-ICPMS analyses served for MT quantification and for evaluation of the MIP/NIP isoform selectivity. MIPs combined with MALDI-MS and LA-ICP-MS techniques enable precise identification and quantification with high mass accuracy, outstanding sensitivity, and relatively fast analysis; showing further prospects in high-throughput testing. For the separation of particular MT isoforms further investigation and optimization of the two mass spectroscopic techniques will be performed together with application of diverse polymers for MIP formation.

\section{Conflicts of interest}

There are no conflicts to declare.

\section{Acknowledgments}

This research has been financially supported by the Czech Science Foundation (project No. 17-12774S), Ministry of Education, Youth and Sports of the Czech Republic under the project CEITEC 2020 (LQ1601) and National Science Center of Poland (NCN) under Sonata Bis grant no. 2012/07/E/NZ1/01894 (to A.K.). Tereza Vaneckova is a Brno Ph.D. Talent Scholarship Holder - funded by the Brno City Municipality. VA wishes to express his thanks to the European Research Council (ERC) under the European Union's Horizon 2020 research and innovation programme (grant agreement No 759585). LPV was supported by project 6SA17676 which received funding from the European Union's Horizon 2020 research and 
innovation programme under the Marie Skłodowska-Curie and which is co-financed by the South Moravian Region under grant agreement No. 665860. 


\section{Figures}

\section{Figure 1}

Overall workflow of molecularly imprinted polymer (MIP) formation, sampling and MALDI-

MS detection of MT-1.

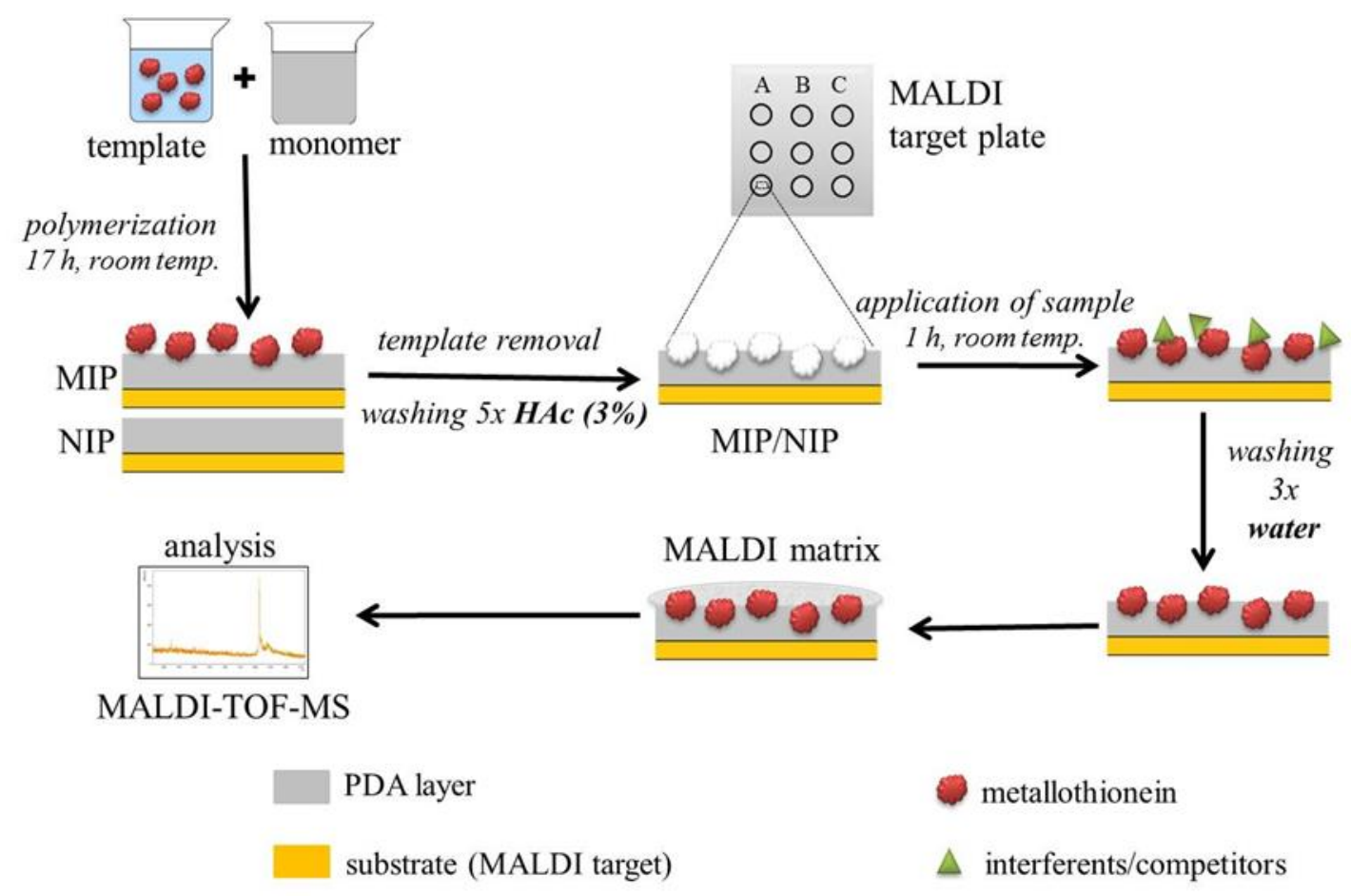




\section{Figure 2}

LA-ICP-MS quantification of ${ }^{66} \mathrm{Zn}$ of different MT-1 concentrations in the sample extracted by MIP (red) and NIP (grey). ${ }^{66} \mathrm{Zn}$ quantification in MIP after template removal is shown in blue.

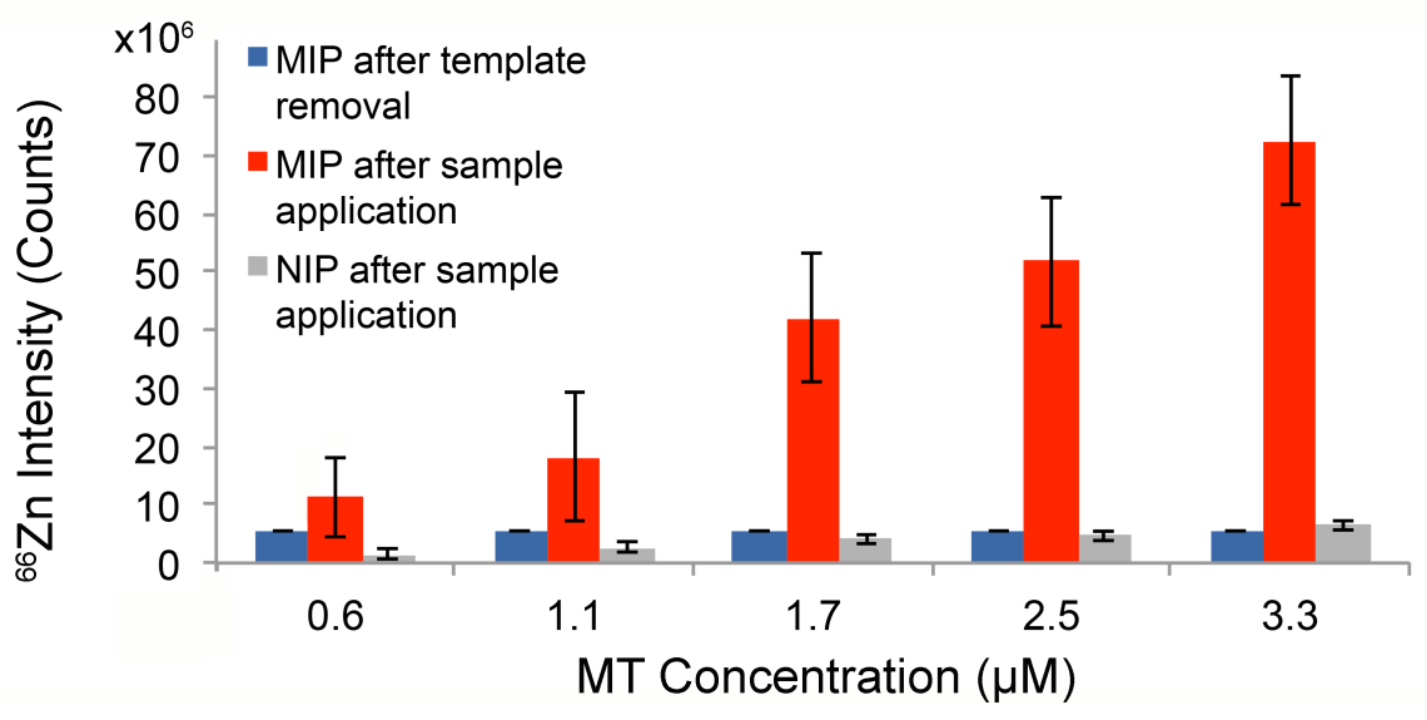




\section{Figure 3}

LA-ICP-MS analyses of MIP and NIP using the MT-1 (Cd) and MT-3 (Zn) templates. (A) Sample containing MT-3(Zn) was applied to MIP/NIP created with template MT-1(Cd). (B) Sample containing MT-1(Cd) was applied to MIP/NIP created with template MT-3(Zn), (C) Sample containing MT-3(Zn) and MT-1(Cd) was applied to MIP/NIP created with template MT-1(Cd).
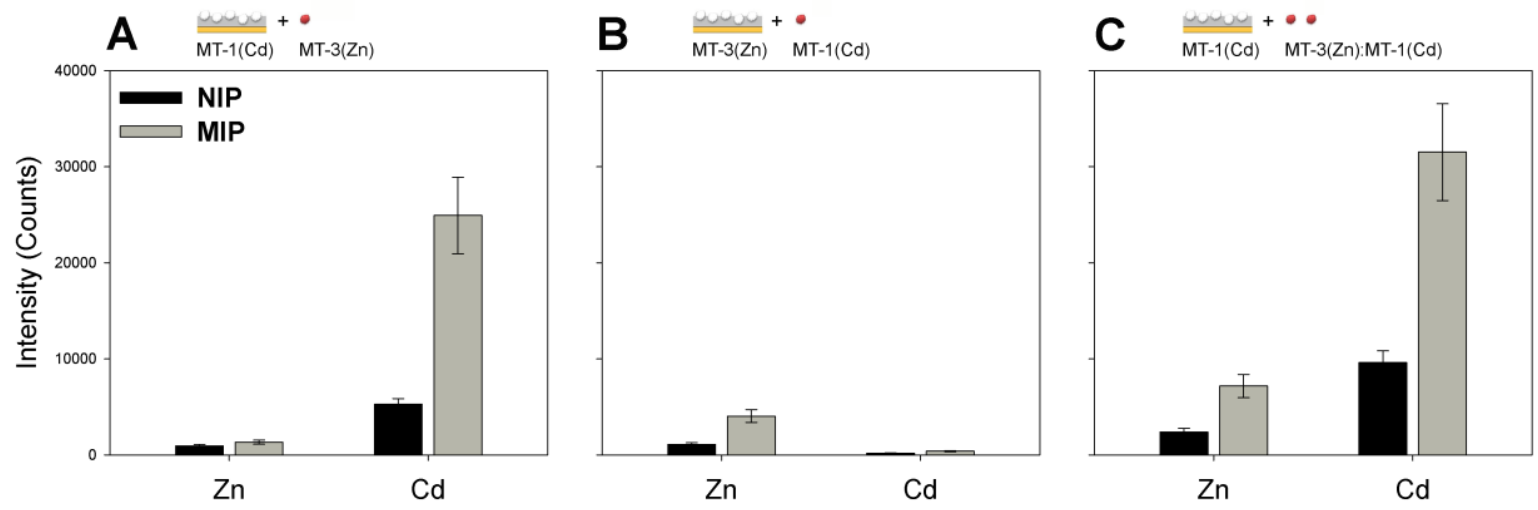


\section{Figure 4}

Stacked overlay of MALDI-TOF-MS data using MT-MIP purification of the plasma spiked with $5 \mu \mathrm{M}$ MT-1 (red), in comparison with NIP purification (blue), MT-1 peak $(\mathrm{m} / \mathrm{z}$ 6071.5) is marked with $\left(^{*}\right)$.

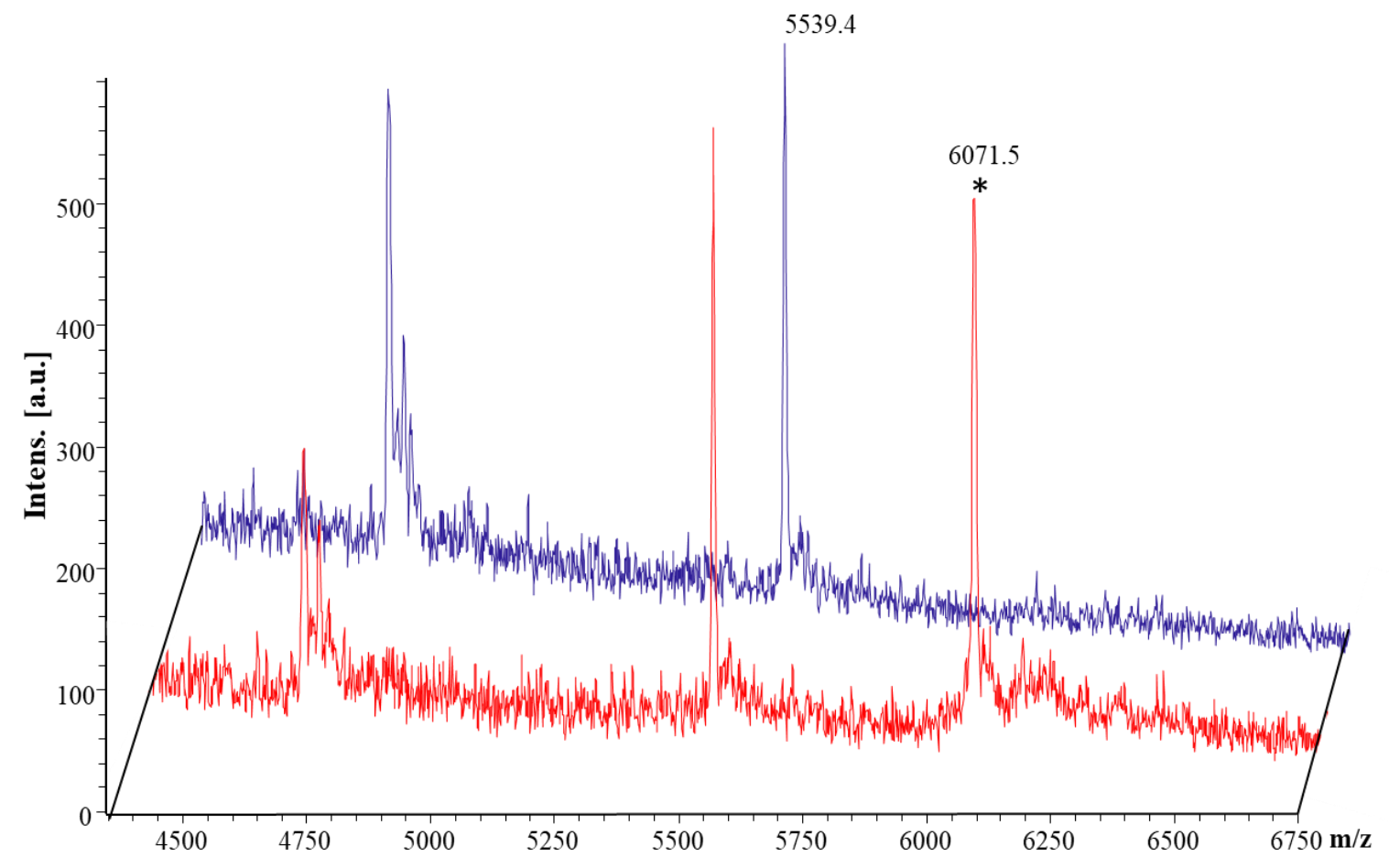




\section{References}

[1] P. Coyle, J.C. Philcox, L.C. Carey, A.M. Rofe, Metallothionein: The multipurpose protein, Cell. Mol. Life Sci. 59(4) (2002) 627-647.

[2] M.A. Dunn, T.L. Blalock, R.J. Cousins, Metallothionein, Proc. Soc. Exp. Biol. Med. 185(2) (1987) 107-119.

[3] D.H. Hamer, Metallothionein, Annu. Rev. Biochem. 55 (1986) 913-951.

[4] A.T. Miles, G.M. Hawksworth, J.H. Beattie, V. Rodilla, Induction, regulation, degradation, and biological significance of mammalian metallothioneins, Crit. Rev. Biochem. Mol. Biol. 35(1) (2000) 35-70.

[5] J.H. Beattie, A.M. Wood, P. Trayhurn, B. Jasani, A. Vincent, G. McCormack, A.K. West, Metallothionein is expressed in adipocytes of brown fat and is induced by catecholamines and zinc, Am. J. Physiol.-Regul. Integr. Comp. Physiol. 278(4) (2000) R1082-R1089.

[6] W. Maret, The function of zinc metallothionein: A link between cellular zinc and redox state, J. Nutr. 130(5) (2000) 1455S-1458S.

[7] B. Ruttkay-Nedecky, L. Nejdl, J. Gumulec, O. Zitka, M. Masarik, T. Eckschlager, M. Stiborova, V. Adam, R. Kizek, The Role of Metallothionein in Oxidative Stress, Int. J. Mol. Sci. 14(3) (2013) 6044-6066.

[8] M. Capdevila, R. Bofill, O. Palacios, S. Atrian, State-of-the-art of metallothioneins at the beginning of the 21st century, Coord. Chem. Rev. 256(1-2) (2012) 46-62.

[9] T. Eckschlager, V. Adam, J. Hrabeta, K. Figova, R. Kizek, Metallothioneins and Cancer, Curr. Protein Pept. Sci. 10(4) (2009) 360-375.

[10] J. Gumulec, M. Raudenska, V. Adam, R. Kizek, M. Masarik, Metallothionein Immunohistochemical Cancer Biomarker: A Meta-Analysis, PLoS One 9(1) (2014) 1-14.

[11] C. Ferrario, P. Lavagni, M. Gariboldi, C. Miranda, M. Losa, L. Cleris, F. Formelli, S. Pilotti, M.A. Pierotti, A. Greco, Metallothionein $1 \mathrm{G}$ acts as an oncosupressor in papillary thyroid carcinoma, Lab. Invest. 88(5) (2008) 474-481.

[12] J. Fu, H.J. Lv, H.X. Guan, X.Y. Ma, M.J. Ji, N.Y. He, B.Y. Shi, P. Hou, Metallothionein $1 \mathrm{G}$ functions as a tumor suppressor in thyroid cancer through modulating the PI3K/Akt signaling pathway, BMC Cancer 13 (2013) 1-13.

[13] M. Kanda, S. Nomoto, Y. Okamura, Y. Nishikawa, H. Sugimoto, N. Kanazumi, S. Takeda, A. Nakao, Detection of metallothionein $1 \mathrm{G}$ as a methylated tumor suppressor gene in human hepatocellular carcinoma using a novel method of double combination array analysis, Int. J. Oncol. 35(3) (2009) 477-483.

[14] M. Ryvolova, S. Krizkova, V. Adam, M. Beklova, L. Trnkova, J. Hubalek, R. Kizek, Analytical Methods for Metallothionein Detection, Curr. Anal. Chem. 7(3) (2011) 243-261. 
[15] V. Adam, I. Fabrik, T. Eckschlager, M. Stiborova, L. Trnkova, R. Kizek, Vertebrate metallothioneins as target molecules for analytical techniques, Trac-Trends Anal. Chem. 29(5) (2010) 409-418.

[16] A. Houessinon, C. Franois, C. Sauzay, C. Louandre, G. Mongelard, C. Godin, S. Bodeau, S. Takahashi, Z. Saidak, L. Gutierrez, J.M. Regimbeau, N. Barget, J.C. Barbare, N. Ganne, B. Chauffert, R. Coriat, A. Galmiche, Metallothionein-1 as a biomarker of altered redox metabolism in hepatocellular carcinoma cells exposed to sorafenib, Mol. Cancer 15 (2016) 110.

[17] L. Nejdl, H.V. Nguyen, L. Richtera, S. Krizkova, R. Guran, M. Masarik, D. Hynek, Z. Heger, K. Lundberg, K. Erikson, V. Adam, R. Kizek, Label-free bead-based metallothionein electrochemical immunosensor, Electrophoresis 36(16) (2015) 1894-1904.

[18] M.A.M. Rodrigo, J. Molina-Lopez, A.M.J. Jimenez, E.P. Del Pozo, P. Adam, T. Eckschlager, O. Zitka, L. Richtera, V. Adam, The Application of Curve Fitting on the Voltammograms of Various Isoforms of Metallothioneins-Metal Complexes, Int. J. Mol. Sci. 18(3) (2017) 1-11.

[19] K. Coufalikova, I. Benesova, T. Vaculovic, V. Kanicky, J. Preisler, LC coupled to ESI, MALDI and ICP MS - A multiple hyphenation for metalloproteomic studies, Anal. Chim. Acta 968 (2017) 58-65.

[20] J.B. Shabb, W.W. Muhonen, A.A. Mehus, Quantitation of Human Metallothionein Isoforms in Cells, Tissues, and Cerebrospinal Fluid by Mass Spectrometry, in: A.K. Shukla (Ed.), Proteomics in Biology, Pt B2017, pp. 413-431.

[21] G. Wulff, Molecular Imprinting in Cross-Linked Materials with the Aid of Molecular Templates - A Way towards Artificial Antibodies, Angew. Chem.-Int. Edit. Engl. 34(17) (1995) 1812-1832.

[22] L.X. Chen, S.F. Xu, J.H. Li, Recent advances in molecular imprinting technology: current status, challenges and highlighted applications, Chem. Soc. Rev. 40(5) (2011) 29222942.

[23] G. Vasapollo, R. Del Sole, L. Mergola, M.R. Lazzoi, A. Scardino, S. Scorrano, G. Mele, Molecularly Imprinted Polymers: Present and Future Prospective, Int. J. Mol. Sci. 12(9) (2011) 5908-5945.

[24] K. Smolinska-Kempisty, A. Guerreiro, F. Canfarotta, C. Caceres, M.J. Whitcombe, S. Piletsky, A comparison of the performance of molecularly imprinted polymer nanoparticles for small molecule targets and antibodies in the ELISA format, Sci. Rep. 6 (2016) 1-7.

[25] A. Bossi, F. Bonini, A.P.F. Turner, S.A. Piletsky, Molecularly imprinted polymers for the recognition of proteins: The state of the art, Biosens. Bioelectron. 22(6) (2007) 11311137.

[26] M. Bertolla, L. Cenci, A. Anesi, E. Ambrosi, F. Tagliaro, L. Vanzetti, G. Guella, A.M. Bossi, Solvent-Responsive Molecularly Imprinted Nanogels for Targeted Protein Analysis in MALDI-TOF Mass Spectrometry, ACS Appl. Mater. Interfaces 9(8) (2017) 6908-6915. 
[27] Y. Chen, D.J. Li, Z.J. Bie, X.P. He, Z. Liu, Coupling of Phosphate-Imprinted Mesoporous Silica Nanoparticles-Based Selective Enrichment with Matrix-Assisted Laser Desorption Ionization-Time-of-Flight Mass Spectrometry for Highly Efficient Analysis of Protein Phosphorylation, Anal. Chem. 88(2) (2016) 1447-1454.

[28] A.N. Tang, L.P. Duan, M.J. Liu, X.C. Dong, An epitope imprinted polymer with affinity for kininogen fragments prepared by metal coordination interaction for cancer biomarker analysis, J. Mat. Chem. B 4(46) (2016) 7464-7471.

[29] R.M. Caprioli, T.B. Farmer, J. Gile, Molecular imaging of biological samples: Localization of peptides and proteins using MALDI-TOF MS, Anal. Chem. 69(23) (1997) $4751-4760$.

[30] W.T. Jia, H.X. Wu, H.J. Lui, N. Li, Y. Zhang, R.F. Cai, P.Y. Yang, Rapid and automatic on-plate desalting protocol for MALD-MS: Using imprinted hydrophobic polymer template, Proteomics 7(15) (2007) 2497-2506.

[31] A. Mathur, S. Blais, C.M.V. Goparaju, T. Neubert, H. Pass, K. Levon, Development of a Biosensor for Detection of Pleural Mesothelioma Cancer Biomarker Using Surface Imprinting, PLoS One 8(3) (2013) 1-8.

[32] F. Sineriz, Y. Ikeda, E. Petit, L. Bultel, K. Haupt, J. Kovensky, D. Papy-Garcia, Toward an alternative for specific recognition of sulfated sugars. Preparation of highly specific molecular imprinted polymers, Tetrahedron 63(8) (2007) 1857-1862.

[33] W. Wan, Q. Han, X.Q. Zhang, Y.M. Xie, J.P. Sun, M.Y. Ding, Selective enrichment of proteins for MALDI-TOF MS analysis based on molecular imprinting, Chem. Commun. 51(17) (2015) 3541-3544.

[34] M. Zhang, X.H. Zhang, X.W. He, L.X. Chen, Y.K. Zhang, A self-assembled polydopamine film on the surface of magnetic nanoparticles for specific capture of protein, Nanoscale 4(10) (2012) 3141-3147.

[35] R.X. Gao, L.L. Zhang, Y. Hao, X.H. Cui, Y.H. Tang, Specific removal of protein using protein imprinted polydopamine shells on modified amino-functionalized magnetic nanoparticles, RSC Adv. 4(110) (2014) 64514-64524.

[36] N.E. Wezynfeld, E. Stefaniak, K. Stachucy, A. Drozd, D. Plonka, S.C. Drew, A. Krezel, W. Bal, Resistance of $\mathrm{Cu}(\mathrm{A}$ beta 4-16) to Copper Capture by Metallothionein-3 Supports a Function for the A beta 4-42 Peptide as a Synaptic Cu-II Scavenger, Angew. Chem.-Int. Edit. 55(29) (2016) 8235-8238.

[37] K. Eersels, P. Lieberzeit, P. Wagner, A Review on Synthetic Receptors for Bioparticle Detection Created by Surface-Imprinting Techniques $\square$ From Principles to Applications, ACS Sensors 1(10) (2016) 1171-1187.

[38] Y.L. Liu, K.L. Ai, L.H. Lu, Polydopamine and Its Derivative Materials: Synthesis and Promising Applications in Energy, Environmental, and Biomedical Fields, Chem. Rev. 114(9) (2014) 5057-5115.

[39] T.P. Chen, T.C. Liu, T.L. Su, J.F. Liang, Self-Polymerization of Dopamine in Acidic Environments without Oxygen, Langmuir 33(23) (2017) 5863-5871. 
[40] W.H. Zhou, C.H. Lu, X.C. Guo, F.R. Chen, H.H. Yang, X.R. Wang, Mussel-inspired molecularly imprinted polymer coating superparamagnetic nanoparticles for protein recognition, J. Mater. Chem. 20(5) (2010) 880-883.

[41] Z.W. Xia, Z.A. Lin, Y. Xiao, L. Wang, J.N. Zheng, H.H. Yang, G.N. Chen, Facile synthesis of polydopamine-coated molecularly imprinted silica nanoparticles for protein recognition and separation, Biosens. Bioelectron. 47 (2013) 120-126.

[42] D.A. Spivak, Optimization, evaluation, and characterization of molecularly imprinted polymers, Adv. Drug Deliv. Rev. 57(12) (2005) 1779-1794.

[43] K.J. Xu, Y.Z. Wang, X.X. Wei, J. Chen, P.L. Xu, Y.G. Zhou, Preparation of magnetic molecularly imprinted polymers based on a deep eutectic solvent as the functional monomer for specific recognition of lysozyme, Microchimica Acta 185(2) (2018) 146-154.

[44] G.H. Yao, R.P. Liang, C.F. Huang, Y. Wang, J.D. Qiu, Surface Plasmon Resonance Sensor Based on Magnetic Molecularly Imprinted Polymers Amplification for Pesticide Recognition, Anal. Chem. 85(24) (2013) 11944-11951.

[45] F. Lan, S.H. Ma, Q. Yang, L.Q. Xie, Y. Wu, Z.W. Gu, Polydopamine-based superparamagnetic molecularly imprinted polymer nanospheres for efficient protein recognition, Colloid Surf. B-Biointerfaces 123 (2014) 213-218.

[46] Y.L. Jeyachandran, E. Mielezarski, B. Rai, J.A. Mielczarski, Quantitative and Qualitative Evaluation of Adsorption/Desorption of Bovine Serum Albumin on Hydrophilic and Hydrophobic Surfaces, Langmuir 25(19) (2009) 11614-11620.

[47] H.M. Chen, C.H. Deng, X.M. Zhang, Synthesis of Fe3O4@SiO2@PMMA Core-ShellShell Magnetic Microspheres for Highly Efficient Enrichment of Peptides and Proteins for MALDI-ToF MS Analysis, Angew. Chem.-Int. Edit. 49(3) (2010) 607-611.

[48] S. Krizkova, M. Ryvolova, J. Gumulec, M. Masarik, V. Adam, P. Majzlik, J. Hubalek, I. Provaznik, R. Kizek, Electrophoretic fingerprint metallothionein analysis as a potential prostate cancer biomarker, Electrophoresis 32(15) (2011) 1952-1961.

[49] A.S. Benk, C. Roesli, Label-free quantification using MALDI mass spectrometry: considerations and perspectives, Anal. Bioanal. Chem. 404(4) (2012) 1039-1056.

[50] A. Krezel, W. Maret, The Functions of Metamorphic Metallothioneins in Zinc and Copper Metabolism, Int. J. Mol. Sci. 18(6) (2017). 